\title{
Platelet-derived or soluble CD154 induces vascularized allograft rejection independent of cell-bound CD154
}

\author{
He Xu, Xiaojie Zhang, Roslyn B. Mannon, and Allan D. Kirk \\ Transplantation Branch, National Institute of Diabetes, Digestive, and Kidney Disease, NIH, United States Department of Health and Human Services, \\ Bethesda, Maryland, USA.
}

\begin{abstract}
CD154 is a cell surface molecule expressed on activated T cells that binds to CD40, an activating molecule on APCs. Its blockade has been shown to prevent allograft rejection, presumably by interrupting interactions between T cells and APCs. It is known that activated human platelets express and shed CD154 and can induce APC activation and other immune processes in vitro. Here we show that platelet-derived CD154 is sufficient to initiate cardiac allograft rejection independent of any cellular source of this molecule. CD154-KO mice reject cardiac allografts after receiving CD154-expressing human platelets or recombinant CD154 (rCD154) trimers. Treatment with the human CD154-specific mAb 5c8 specifically prevents this induced rejection. Soluble trimers, but not platelets, induce rejection when infused temporally remote from the surgical procedure, suggesting that surgically induced platelet activation is required for CD154 release. Allograft rejection can thus be instigated by activated platelets through CD154. These data implicate platelets as a proximal component of acquired alloimmunity, providing insight into the mechanisms of allograft rejection and the physiological response to trauma in general.
\end{abstract}

\section{Introduction}

CD154 is a 33-kDa type II transmembrane protein originally identified on activated $T$ cells as the ligand for CD40, a molecule promoting B cell and APC activation (1). CD154 has been shown to play a critical role in the generation of alloimmune responses. Specifically, treatment with CD154-specific mAbs prevents acute allograft rejection in both murine and nonhuman primate models, presumably by blocking T cell interactions with APCs expressing CD40 (2-7). However, CD154-directed therapy has also been shown to require high levels of antibody to achieve an effect, greatly exceeding those typically required of antibodies targeting $\mathrm{T}$ cell activation markers (8). Furthermore, unlike antibody therapy directed toward $\mathrm{T}$ cell molecules, treatment with CD154-specific antibodies has been associated with thromboembolic complications (9).

It has been recently shown that human platelets contain preformed CD154 and express and shed this molecule upon activation (10). Both cell-surface-bound and soluble forms of CD154 exist as homotrimers $(11,12)$, and soluble platelet-derived CD154 has been shown to mediate endothelial and APC activation in vitro $(10,13-15)$. Indeed, CD154 cleaved and released in soluble form after platelet activation has been considered a major source of circulating CD154 (16). Furthermore, soluble CD154 has increasingly been associated with adverse inflammatory conditions in humans, including lupus and acute coronary syndrome $(17,18)$. To date, however, there has been no direct evidence in vivo that soluble CD154 can mediate pathological immune responses independent of cell-bound CD154.

Organ transplantation is, by necessity, associated with surgical trauma. Given that biologically active CD154 is present in platelets

Nonstandard abbreviations used: rCD154, recombinant CD154; MLR, mixed lymphocyte reaction.

Conflict of interest: The authors have declared that no conflict of interest exists. Citation for this article: J. Clin. Invest. doi:10.1172/JCI27155. and released upon activation and that platelet activation is unavoidable in any surgical procedure, we have hypothesized that platelet activation induced during organ transplantation contributes significantly to allograft rejection through the CD154 pathway (14, $19,20)$ and that soluble CD154 significantly influences the efficacy of CD154-targeted therapies. We have thus explored the role of non-T cell-derived CD154 in vascularized allograft rejection to determine if it induces rejection in vivo. We found that human platelet-derived or soluble recombinant CD154 (rCD154) induces cardiac allograft rejection independent of any cell-bound source of this molecule and that platelets can initiate alloimmunity through CD154 release when activated at the time of a surgical procedure.

\section{Results}

Human CD154 is biologically active in a murine environment. We first determined whether human CD154 had biological activity in mice such that cell-bound and soluble forms of the human molecule could be clearly distinguished and independently studied in vivo. We evaluated 1-way mixed lymphocyte reactions (MLRs) using WT C57BL/6 or CD154-KO (B6.129S2-Tnfsf5) splenocytes as responders and fully $\mathrm{MHC}$-mismatched $\mathrm{BALB} / \mathrm{c}$ splenocytes as stimulators in the presence or absence of human rCD154 trimers. As expected, CD154-KO splenocytes had attenuated proliferative responses to BALB/c splenocytes compared with WT splenocytes, and CD154 blockade with a murine CD154-specific mAb, MR-1, inhibited proliferation of WT splenocytes (data not shown). The addition of human rCD154 trimers induced dose-dependent proliferation of CD154-KO splenocytes but only when concomitantly stimulated by BALB/c splenocytes (data not shown). This proliferative response was not inhibited by MR-1, which is only specific for murine CD154, but was inhibited by the human-specific mAb $5 c 8$ (Figure 1). Isotype control antibodies for both MR-1 and 5c8 did not block lymphocyte proliferation, and the human rCD154 trimers, when incubated with CD154-KO murine splenocytes 


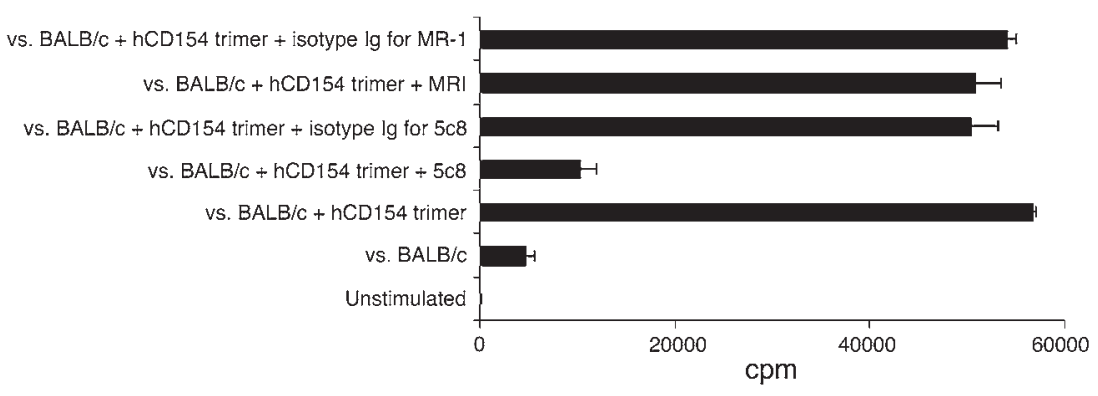

Figure 1

Human CD154 (hCD154) induces cellular proliferation in mice that is blocked by human CD154-specific but not murine CD154-specific antibodies. Shown are results from MLR using B6-CD154-KO responders (B6.129S2-Tnfsf5) versus BALB/c stimulators with or without human rCD154 trimers $(50 \mu \mathrm{g} / \mathrm{ml})$ and/or the human- or murine-specific CD154 mAbs. $\mathrm{KO}$ lymphocytes respond poorly in MLRs, and this response is greatly augmented by the addition of human CD154 trimers. This proliferation is specifically blocked by the humanspecific antibody $5 \mathrm{c} 8$ but not by the murine-specific antibody MR-1 or isotype controls.

without allogeneic antigens, induced minimal lymphocyte proliferation (data not shown). CD154-KO spleens were evaluated by flow cytometry and shown to have ample numbers of CD4- and CD8-positive T cells. Thus, the attenuated response of CD154$\mathrm{KO}$ splenocytes was not attributable to relative $\mathrm{T}$ cell deficiency in these mice.

To further define the extent to which human CD154 could influence murine responses, we evaluated human CD154 trimer interactions with murine DCs. We stimulated murine DCs, isolated from B6.129S7-Rag1 mice, with human rCD154 trimers in the presence or absence of recombinant murine IFN- $\gamma$ for 24 hours. The supernatants were collected and analyzed by ELISA for murine IL-12 (p70), an indicator of DC activation. LPS was used as a positive control. Human CD154 trimers induced production of IL-12, an effect that was further augmented by the presence of murine IFN- $\gamma$ (data not shown). These results indicate that soluble human CD154 trimers sufficiently cross-react with murine CD40 to initiate allogeneic cell-mediated immune responses. Thus, human soluble CD154 could be investigated independent of any cell-bound sources of CD154 in a murine system.

Since it has been shown that human CD154 can, in addition to activating APCs, also activate human ECs, we also sought to determine if human CD154 activated murine endothelium. Cultured murine EC monolayers were stimulated with human CD154 trimers at $100 \mu \mathrm{g} / \mathrm{ml}$ for 2, 4, and 24 hours followed by FACS analysis for CD54 and CD62E. Recombinant murine TNF- $\alpha$ served as a positive control. Human CD154 trimers stimulation did not lead to upregulation of CD54 beyond its basal expression (data not shown) and did not lead to CD62E expression (Figure 2). Thus, human CD154 activates murine APCs but not murine ECs, and its effects are thus indicative of immune activation rather than changes in $\mathrm{EC}$ adhesion.

Soluble CD154 initiates vascularized allograft rejection. To evaluate the ability of soluble CD154 to initiate allograft rejection, the effects of human rCD154 trimers were tested in vivo in a well-established murine heterotopic cardiac transplant model (21). C57BL/ 6 recipients $(n=10)$ rejected BALB/c cardiac allografts in a mean of 8.2 days (Figure $3 \mathrm{~A}$ ). Treatment with a single $1 \mathrm{mg}$ dose of MR-1 prevented acute cardiac allograft rejection for more than 80 days $(n=10$; Figure 3A). CD154-KO recipients similarly experienced long-term cardiac allograft survival with $80 \%$ of the animals surviving for more than 100 days $(n=14$; Figure $3 \mathrm{~A})$. In contrast, when CD154-KO animals received a single intravenous infusion of human rCD154 trimers $(500 \mu \mathrm{g})$ at the time of transplantation, 11 of 12 rejected their allografts on postoperative days 8 to $34(P<0.001$ versus untreated $\mathrm{KO}$ animals; Figure 3A). Additionally, this trimer-induced rejection was prevented in 5 of 5 CD154-KO recipients by treatment with the human-CD154-specific antibody $5 \mathrm{c} 8$ (200 $\mu \mathrm{g}$ on days $-1,0,3,5$, and 7$)$, initiated before trimer infusion $(P=0.002$ versus trimertreated KO animals; Figure 3A). Trimer injection into WT animals significantly decreased the time to rejection from a mean of 8.2 days to a mean of 6.8 days $(n=6 ; P=0.001$; Figure 3A).

Histological evaluation of the rejected cardiac allografts showed acute cellular rejection with a typical lymphocyte infiltration and vascular endothelial cell activation both in WT untreated animals (data not shown) and in CD154-KO recipients that received rCD154 trimers (Figure 3B). In contrast, cardiac allografts harvested from CD154-KO recipients treated with CD154 trimers and $5 \mathrm{c} 8$ showed no evidence of acute rejection even 100 days after transplantation (Figure 3C). These observations demonstrate that a soluble form of human CD154 can initiate cardiac allograft rejection without any cell-bound source of this molecule.

Platelets can serve as the sole source of CD154 in allograft rejection. To directly test whether CD154 released in response to surgical trauma initiates allograft rejection, we obtained purified resting human platelets and verified the absence of CD154 in a resting state and their ability to express CD154 following stimulation by human thrombin or thrombin receptor activator peptide (TRAP-1) (data not shown). To evaluate the fate of human platelets in mice, human platelets were labeled with PKH-26 and then
Figure 2

Soluble rCD154 trimers $(100 \mu \mathrm{g} / \mathrm{ml})$ do not activate murine vascular ECs. Shown are flow cytometry analyses of murine ECs stained for CD62E following stimulation with TNF- $\alpha$ (a positive control) or human rCD154.
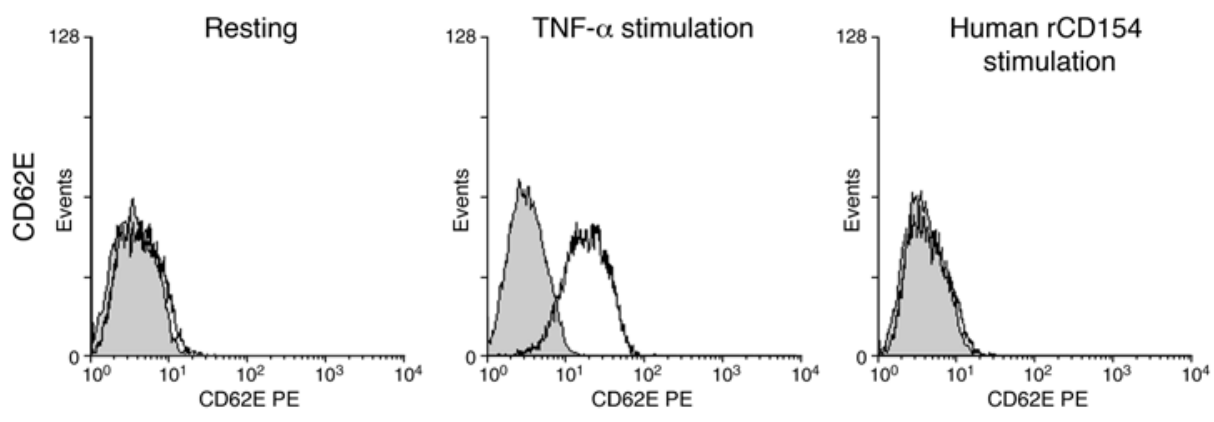


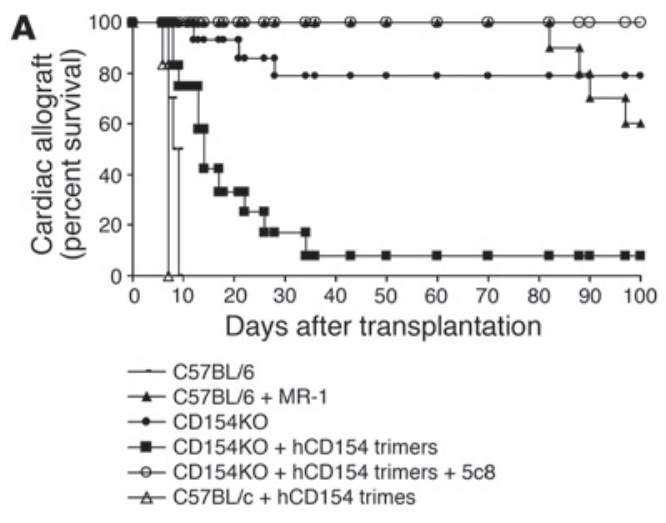

B

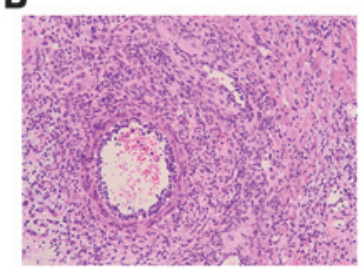

C

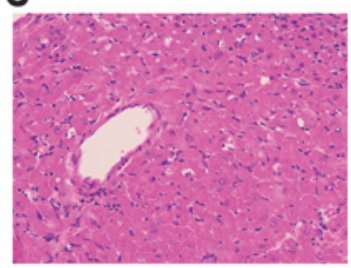

adoptively transferred into CD154-KO mice via tail vein injection. Flow cytometry demonstrated that platelets were cleared from the circulation within 24 hours (data not shown). Histological evaluation of spleens collected from these mice revealed PKH-26-positive platelets located predominantly in the splenic red pulp within the first 24 hours (Figure 4A) that persisted for at least 14 days (data not shown). Platelets were also detected in the interstitium of the allograft 1 day after transplantation (Figure 4A) and to a lesser degree in the periaortic lymph nodes (data not shown).

We then adoptively transferred resting human platelets into CD154-KO recipients $(n=28)$ followed by cardiac allografts from $\mathrm{BALB} / \mathrm{c}$ donors. Half of these recipients were also treated with the human CD154-specific mAb 5c8 (200 $\mu$ g on days $-1,0,3$,

\section{Figure 4}

CD154-deficient mice reject cardiac allografts following human platelet transfusion. This is blocked by human CD154-specific 5c8. (A) $\mathrm{PKH}-26-$ labeled human platelets aggregate in the splenic red pulp and are also seen in the interstitium of the transplanted heart 24 hours following intravenous infusion. Shown is an H\&E-stained section and its corresponding fluorescence image for spleen and cardiac allograft tissue derived from a mouse following injection of $\mathrm{PKH}-26$-stained platelets. (B) CD154-KO recipients $(n=28)$ received human platelet transfusions followed by cardiac allografts; half were treated with $5 \mathrm{c} 8$. Animals that received platelets rejected $(P=0.008$ versus nontransfused animals), but those pretreated with $5 \mathrm{c} 8$ did not $(P=0.012$ versus untreated platelet-transfused animals). Infusion of platelets into WT animals $(n=5)$ accelerated the rate of rejection by 0.8 days $(P=0.057)$. Control groups C57BL/6, C57BL/6 + MR-1, and CD154KO represent the same animals described in the Figure 3 legend and are displayed in this graph for comparative purposes. (C) Cardiac allograft rejection in CD154-KO recipients that received human platelet transfusions. Magnification, $\times 20$. (D) Allografts harvested from CD154-KO recipients treated with anti-CD154 5c8 followed by infusion of human platelets are protected from rejection and are histologically similar to cardiac allografts collected from untreated CD154-KO recipients that do not reject (not shown). Magnification, $\times 20$.

\section{Figure 3}

Soluble rCD154 trimers induce cardiac allograft rejection in CD154deficient mice. (A) C57BL/6 recipients accept BALB/c donor heart allografts with $(n=10)$ but not without $(n=10)$ CD154 blockade using MR-1. Similarly, CD154-KO mice accept cardiac allografts $(n=14)$. However, the infusion of human rCD154 trimers $(500 \mu \mathrm{g})$ induces rejection by CD154-KO recipients $(n=12 ; P<0.001$ versus untreated animals). Human CD154 blockade with the anti-human CD154 antibody $5 \mathrm{c} 8$ restores the tolerant phenotype to animals receiving CD154 trimers ( $n=5 ; P=0.002$ versus trimer-treated animals). Infusion of trimers into WT animals $(n=6)$ accelerated the rate of rejection by an average of 1.4 days ( $P=0.001$ versus untreated controls). (B) Cardiac allografts from CD154-KO recipients that received human rCD154 trimers show acute cellular rejection. Magnification, $\times 20$. (C) Allografts from CD154-KO recipients treated with $5 \mathrm{c} 8$ followed by infusion of human rCD154 trimers show normal myocardium without rejection. Magnification, $\times 40$.

5, and 7). Ten of $14 \mathrm{CD} 154-\mathrm{KO}$ recipients that received human platelets promptly rejected their cardiac allografts $(P=0.008$ versus untreated $\mathrm{KO}$ animals; Figure $4 \mathrm{~B}$ ), with histological findings that were indistinguishable from control rejecting animals (Figure 4C). Treatment with $5 c 8$ prevented cardiac allograft rejection in all animals $(P=0.012$ versus platelet-treated $\mathrm{KO}$ animals; Figure $4 \mathrm{~B})$. At sacrifice, these animals had no histological evidence of acute rejection (Figure 4C), and their histology was similar to that seen in CD154-KO recipients that did not receive trimers or platelet infusions. As with trimer infusion, infusion of human platelets

A
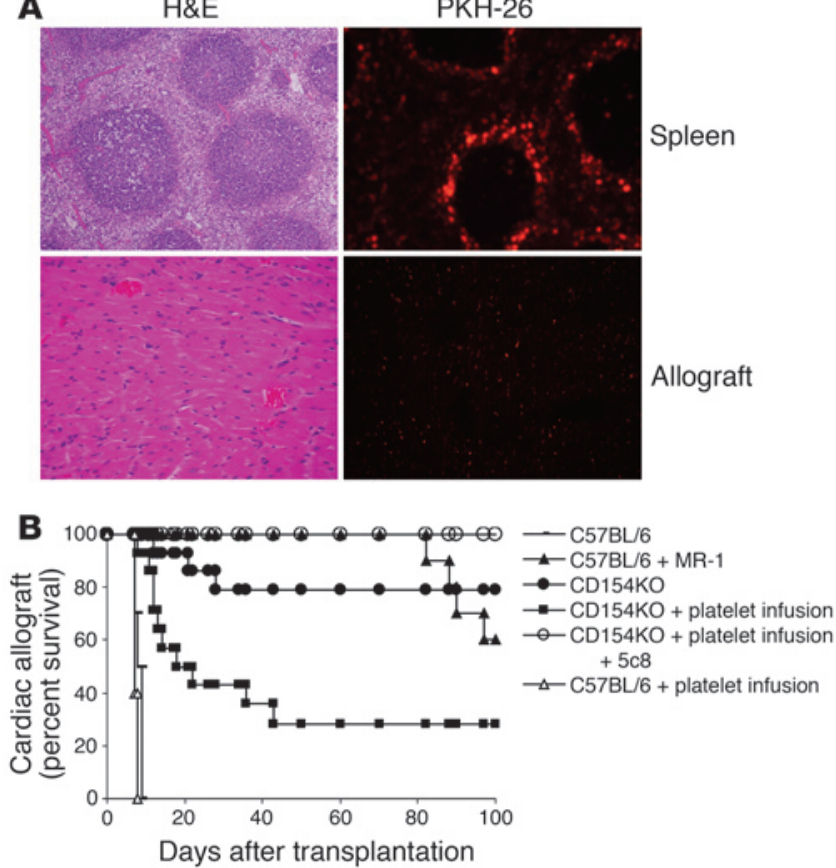

C

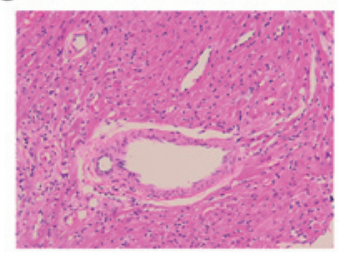

D

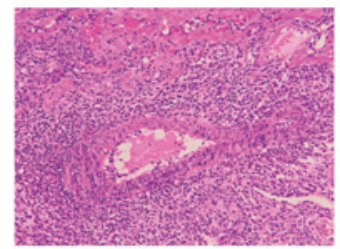



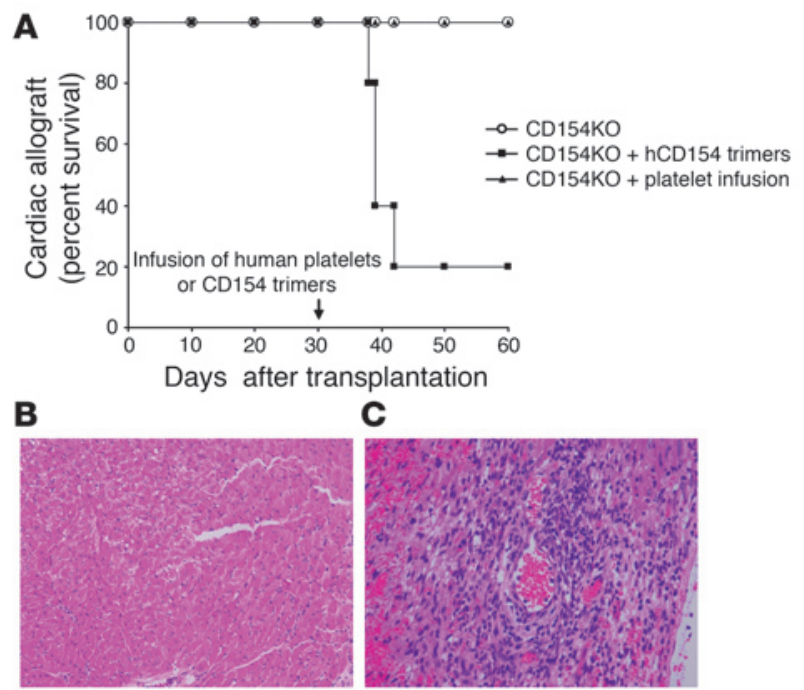

Figure 5

When infused 30 days after surgery, soluble rCD154 induces rejection but platelets do not. (A) CD154KO animals that received rCD154 trimers 30 days after transplantation promptly rejected their allografts ( $n=5 ; P=0.008$ versus untreated animals). Animals receiving platelet transfusions 30 days after transplantation did not reject $(n=5)$. (B) CD154-KO recipients that received human platelet transfusions on day 30 were euthanized 30 days after infusion and showed no evidence of rejection. Magnification, $\times 20$. (C) Those receiving trimers had histologically typical rejection. Magnification, $\times 40$.

into WT recipients modestly accelerated the pace of acute rejection to a mean of 7.4 days $(n=5 ; P=0.057$; Figure $4 \mathrm{~B})$.

To determine the role of surgical trauma in activating platelets, CD154-KO animals $(n=14)$ were given cardiac allografts. The animals were allowed to heal for 30 days, at which time platelets were adoptively transferred $(n=5)$ or CD154 trimers were infused $(n=5)$. Mice $(n=4)$ without platelet or trimer infusion were also studied 1 month after transplant for baseline histological evaluation. Unlike those animals that received platelet transfusions at the time of surgery, animals receiving platelet infusions 30 days after transplantation maintained stable allograft function (Figure 5A). These animals were sacrificed at days 30 and 60 after platelet infusion, and histological evaluation showed no evidence of rejection (data not shown). In contrast, 4 of 5 mice receiving CD154 trimer infusions 1 month after cardiac transplantation rejected their allografts within 8 to 12 days $(P=0.008$ versus untreated animals; Figure 5, A and B). The control mice without human platelet or CD154 trimer infusion were euthanized at posttransplant day 30 and did not have rejection (Figure 5C). Thus, soluble CD154 can induce rejection late after transplantation, but platelets are unable to provide sufficient CD154 in the absence of a concomitant activating event such as surgery or reperfusion.

\section{Discussion}

Our data demonstrate for the first time, to our knowledge, that human soluble CD154 is capable of inducing a pathological immune response in vivo, specifically allograft rejection, independent of any cell-bound source of this critical costimulatory molecule. Furthermore, we have shown that human platelets activated in vivo at the time of transplantation induce rejection through CD154. Given the inseparable relationship between surgical trauma and organ transplantation, these findings highlight a novel and potentially important mechanism for alloimmune response initiation and suggest a more general relationship between CD154 and other detrimental posttraumatic immune responses. These findings are relevant to the transition of CD154based therapies into clinical practice and suggest new therapeutic avenues for their application.

CD40 has been firmly established as a critical molecule mediating professional (e.g., monocyte, DC) and opportunistic (e.g., EC) APC function (22-26). The ability of platelet-derived CD154 to mediate these events as well as effector antibody and T cell maturation has become more recently apparent $(10,12-14)$. The current study underscores the importance of this pathway in a clinically relevant pathological immune response, organ rejection, and highlights the considerable potential for platelets to participate in other posttraumatic immune responses, both protective and detrimental. Although the controlled circumstance of an elective surgical procedure is not typically viewed as traumatic, CD154 provides a mechanism for immune activation to be stirred proportional to the injury sustained. Indeed, platelet/monocyte complexes have recently been shown to form following elective surgical procedures (27), and there is a known association between worsening ischemic insult and increased rejection rate (28). The accelerated rejection seen when trimers or platelets were added to WT grafts in this study demonstrates that soluble CD154 can be immunostimulatory even in the WT setting and augment immunity in a manner proportionate to its release. Thus, while it is clear that immune responses such as allograft rejection are not solely predicated on trauma (29), the concept of injury as a driving force behind augmented immunity is easily reconciled with the present findings.

The findings from these studies do not negate the importance of cell-bound CD154 in mediating immune responses. Clearly, CD154 expression on activated T cells influences immune responsiveness through its effects on APCs and also perhaps through direct effects on the $T$ cell itself $(30,31)$. However, they do add considerably to the magnitude of immune induction possible under certain circumstances. Although CD154 is an inducible molecule on the cell surface, the magnitude of its induction through $\mathrm{T}$ cell activation is fixed by the potential number of responding antigen-specific $T$ cells and the rate and degree to which these cells are activated after antigen presentation. Soluble CD154 derived from platelets potentially provides a considerable amount of this ligand independent of and prior to antigen presentation and cell activation. Thus, acquired immunity can be initiated and driven in response to clinical situations such as vascular injury or other traumatic conditions.

It remains unclear if there is a dominant anatomical site in which soluble CD154 mediates its effects. Clearly, the spleen showed the highest concentration of infused platelets in our model, although platelets were also detected in the allograft and to a lesser degree in periaortic lymph nodes. The distribution of trimers is presumably similar to other large macromolecules and is likely broader. The spleen is a known site for alloimmune activation following primarily vascularized transplantation (32), and the effects seen in our study could be explained by augmented antigen presentation in the spleen. However, platelets are intravascularly ubiquitous, and cogent arguments can be made for local CD154 in the graft-stimulating passenger donor APCs and migratory monocytes. Since primarily vascularized allografts have disrupted lymphatic drainage, 
it is difficult to determine the role of a true draining lymph-node bed for most organ grafts, although activated platelets could have a role in nodal tissue as well. We favor a primary role for the spleen during systemic activation as would be expected following a major surgical procedure and are investigating the local and systemic effects of activated platelets.

Based on these data, it is evident that for CD154 blockade to effectively prevent an immune response in a traumatic condition, it must address a potentially large reservoir of platelet-derived CD154. Indeed, in dose-range studies, exceptionally large doses of $\mathrm{mAb}$ have been necessary to prevent rejection in nonhuman primates (8). Given the substantial concern regarding thromboembolic complications arising from the initial clinical trials with antiCD154 $(9,13,33)$, the effects of CD154 cross-linking on platelets will need to be cautiously examined. It may be more feasible to target CD40, a molecule with more limited distribution. Regardless, this non-T cell component must be considered in the design of antirejection therapies and should be scrutinized in other posttraumatic immune responses.

\section{Methods}

Mice. We purchased 6- to 8-week-old male B6.129S2-Tnfsf5 and B6.129S7Rag1 mice from Jackson Laboratory. Eight-week-old BALB/c and C57BL/6 mice were obtained from the National Cancer Institute. The experiments described in this study were conducted according to the principles set forth in the Guide for the care and use of laboratory animals (Institute of Laboratory Animals Resources, National Research Council, Department of Health and Human Services, NIH Pub. No. 86-23, 19850). All procedures were performed according to animal care and use protocols approved by the Intramural Institutional Animal Care and Use Committee of the National Institute of Diabetes, Digestive and Kidney Disease.

Cellular assays. Splenocytes were isolated and MLRs were performed using $2 \times 10^{5}$ responders and mitomycin C-treated stimulators at 3 different ratios to responders or $2 \times 10^{5}$ stimulators. MLRs were carried out for 5 days and pulsed with ${ }^{3} \mathrm{H}$-thymidine at $1 \mu \mathrm{Ci}$ /well during the final 24-hour culture. The lymphocyte proliferation was determined as cpm by ${ }^{3} \mathrm{H}$-thymidine incorporation, using a $\beta$-liquid scintillation counter. The anti-human $\mathrm{CD} 154 \mathrm{mAb} 5 \mathrm{c} 8$ was used at $100 \mu \mathrm{g} / \mathrm{ml}$ for human CD154 blockade. The anti-murine CD154 mAb MR-1 was used at $100 \mu \mathrm{g} / \mathrm{ml}$ for murine CD154 blockade. Isotype control antibodies were hamster IgG (CALTAG Laboratories) and mouse IgG1 (Ancell Corp.).

To evaluate DC activation, murine bone marrow was harvested from the B6.129S7-Rag1 mouse (femur, tibia, and humerus) by flushing the marrow with RPMI-1640 medium, using a 6-ml syringe and 26G needle. Cells were washed after lysing red blood cells and incubated with culture medium containing 10\% FCS, $10 \mathrm{ng} / \mathrm{ml}$ murine granulocyte/macrophage colony stimulating factor, and $10 \mathrm{ng} / \mathrm{ml} \mathrm{IL-4}$ (PeproTech) for 4 days. Nonadherent cells were collected and verified as DCs by positive staining for CD83, CD86, and HLA-DR (> 90\%). DCs $\left(5 \times 10^{4}\right)$ were added into 96-well flatbottom plates in the presence or absence of human rCD154 trimers (50 $\mu \mathrm{g} / \mathrm{ml}$; R\&D Systems) or in combination with recombinant murine IFN- $\gamma$ ( $2 \mathrm{ng} / \mathrm{ml}$; PeproTech). Supernatants were collected after 24-hour stimulation and analyzed by ELISA to detect IL-12 production. DCs stimulated by LPS (60 ng/ml; Sigma-Aldrich) or without stimulation were used as positive and negative controls, respectively. The mouse IL-12 (p70) ELISA kit was obtained from BD Biosciences. An ELISA was performed according to the manufacturer's instructions. The absorbance of each well was measured at $450 \mathrm{~nm}$ within 30 minutes of stopping reaction.

To evaluate direct effects of human CD154 trimers on murine ECs, the $\mathrm{BALB} / \mathrm{c}$ bEnd.3 EC cell line was obtained from ATCC and maintained with Dulbecco's modified Eagle's medium (Invitrogen Corp.) supplemented with $10 \%$ FCS. For positive control activation, ECs were treated with TNF- $\alpha$, and activation was verified by upregulation of CD54 and CD62E as determined by FACS using a FACScan (BD). Murine EC monolayers were stimulated with human CD154 trimers at $100 \mu \mathrm{g} / \mathrm{ml}$ at different time points followed by staining with mAbs directed to either CD62E or CD54 on ice for 30 minutes. Cells were washed twice with FACS buffer followed by incubation with PBS (Invitrogen Corp.) containing $20 \mathrm{mM}$ HEPES (BioWhittaker Inc.), pH 7.4, 10 mM EDTA, and 0.5\% BSA (SigmaAldrich) at $4^{\circ} \mathrm{C}$ for 20 minutes and then at $37^{\circ} \mathrm{C}$ for 20 minutes. Detached ECs were analyzed by FACScan.

Platelet preparation and stimulation. Volunteers were enrolled in an NIH Institutional Review Board-approved tissue acquisition protocol and donated after informed consent was obtained. Purified human platelets were obtained from leukapheresis products from normal volunteers from the Department of Transfusion Medicine, NIH. Platelets were washed twice with Ca2/Mg2-free PBS containing 100 nM PGE-1 (Sigma-Aldrich) and $1.9 \mathrm{mM}$ theophylline by centrifugation. Cells were diluted with PBS to $500 \times 10^{6} / \mathrm{ml}$ and incubated with $100 \mathrm{U} / \mathrm{ml}$ thrombin receptor activator peptide (TRAP) at room temperature for 10 minutes. Both resting and activated platelets were then stained with mouse IgG1-PE, anti-CD41a-PE, anti-CD62P-PE, and anti-CD154-PE mAbs at $4^{\circ} \mathrm{C}$ for 30 minutes followed by flow cytometric analysis.

To detect the presence of human platelets in CD154-KO mice following adoptive transfer, human platelets were labeled with PKH-26, a fluorescent compound that incorporates aliphatic reporter molecules into the cell membrane by selective partitioning, according to the protocol suggested by the manufacturer (Sigma-Aldrich). PKH-26-labeled platelets were evaluated by flow cytometric analysis before transfusion, and peripheral blood was evaluated up to 24 hours after infusion. Spleens, periaortic lymph nodes, and allografts were evaluated histologically 1 day after injection using $\mathrm{H} \& \mathrm{E}$ stains for general architecture and fluorescence microscopy to detect PKH-26-stained platelets. The spleens from nontransplanted animals were also evaluated at 4,8 , and 14 days after injection.

Cardiac transplants and treatments. Transplants were performed heterotopically with vascularized BALB/c cardiac allografts as described using standard microvascular techniques (21). Cardiac allografts were monitored by daily palpation and removed for histological evaluation after cessation of allograft contractility. Human CD154 trimers were injected intravenously via tail vein before cardiac allografts. On days $-1,0,3,5$, and 7, $200 \mu \mathrm{g}$ $5 c 8$ or MR-1 was injected i.p. Graft survival times were compared between groups using 2-tailed unpaired Student's $t$ tests with $P<0.05$ considered as statistically significant.

\section{Acknowledgments}

The authors gratefully acknowledge Frank Leopardi for his expert technical assistance, Mark St. Clair for his veterinary care, and Ashish Jain for his critical reading of this manuscript. This work was funded by the Intramural Research Program of the National Institute of Diabetes and Digestive and Kidney Diseases of the NIH.

Received for publication October 18, 2005, and accepted in revised form January 3, 2006.

Address correspondence to: Allan D. Kirk, Room 5-5752, Building 10CRC, Center Drive, Transplantation Branch, National Institute of Diabetes, Digestive and Kidney Disease, NIH, United States Department of Health and Human Services, Bethesda, Maryland 20892, USA. Phone: (301) 496-3047; Fax: (301) 451-6989; E-mail: allanK@intra.niddk.nih.gov. 
1. Armitage, R.J., et al. 1992. Molecular and biological characterization of a murine ligand for CD40. Nature. 357:80-82.

2. Parker, D.C., et al. 1995. Survival of mouse pancreatic islet allografts in recipients treated with allogeneic small lymphocytes and antibody to CD40 ligand. Proc. Natl. Acad. Sci. U. S. A. 92:9560-9564.

3. Larsen, C.P., et al. 1996. Long-term acceptance of skin and cardiac allografts after blocking CD40 and CD28 pathways. Nature. 381:434-438.

4. Hancock, W.W., et al. 1996. Costimulatory function and expression of CD40 ligand, CD80, and CD86 in vascularized murine cardiac allograft rejection. Proc. Natl. Acad. Sci. U. S. A. 93:13967-13972.

5. Kirk, A.D., et al. 1997. CTLA-4-Ig and anti-CD40 ligand prevent renal allograft rejection in primates. Proc. Natl. Acad. Sci. U. S. A. 94:8789-8794.

6. Kirk, A.D., et al. 1999. Treatment with humanized monoclonal antibody against CD154 prevents acute renal allograft rejection in nonhuman primates. Nat. Med. 5:686-693.

7. Xu, H., et al. 2003. Studies investigating pretransplant donor-specific blood transfusion, rapamycin, and the CD154-specific antibody IDEC-131 in nonhuman primate model of skin allotransplantation. J. Immunol. 170:2776-2782.

8. Xu, H., et al. Effects of dose and duration of antiCD154 antibody therapy in preventing renal allograft rejection in a nonhuman primate model Transplant. Proc. 33:223-224

9. Kawai, T., Andrews, D., Colvin, R.B., Sachs, D.H. and Cosimi, A.B. 2000. Thromboembolic complications after treatment with monoclonal antibody against CD40 ligand [letter]. Nat. Med. 6:114.

10. Henn, V., et al. 1998. CD40 ligand on activated platelets triggers an inflammatory reaction of endothelial cells. Nature. 391:591-594.

11. Pietravalle, F., et al. 1996. Human native soluble CD40L is a biologically active trimer, processed inside microsomes. J. Biol. Chem. 271:5965-5967.
12. Hsu, Y.M., et al. 1997. Heteromultimeric complexes of CD40 ligand are present on the cell surface of human T lymphocytes. J. Biol. Chem. 272:911-915.

13. Elzey, B.D., et al. 2003. Platelet-mediated modulation of adaptive immunity. A communication link between innate and adaptive immune compartments. Immunity. 19:9-19.

14. Czpiaga, M., Kirk, A.D., and Lekstrom-Himes, J. 2004. Platelets deliver costimulatory signals to antigen-presenting cells: a potential bridge between injury and immune activation. Exp. Hematol. 32:135-139.

15. Xu, H., et al. 2001. Human platelets activate porcine endothelial cells through a CD154-dependent pathway. Transplantation. 72:1858-1861.

16. Andre, P. et al. 2002. CD40L stabilizes arterial thrombi by a $\beta 3$ integrin-dependent mechanism. Nat. Med. 8:247-252.

17. Kato, K., et al. 1999. The soluble CD40 ligand sCD154 in systemic lupus erythematosus. J. Clin. Invest. 104:947-955.

18. Heeschen, C., et al. 2003. CD40 ligand in acute coronary syndromes. N. Engl. J. Med. 348:1104-1111.

19. Kirk, A.D. 1999. Let's blame the little guys: platelets as an instigator of allograft rejection? Graft. 2:159-160.

20. Kirk, A.D, Blair, P.J., Tadaki, D.K., Xu, H., and Harlan, D.M. 2001. The role of CD154 in organ transplant rejection and acceptance. Philos. Trans. R. Soc. Lond. B. Biol. Sci. 356:691-702.

21. Corry, R.J., Winn, H.J., and Russell, P.S. 1973. Primary vascularized allograft of hearts in mice. The role of $\mathrm{H}-2 \mathrm{D}, \mathrm{H} 2-\mathrm{K}$, and non-H-2 antigens in rejection. Transplantation. 16:343-350.

22. Alderson, M.R., et al. 1993. CD40 expression by human monocytes: regulation by cytokine and activation of monocytes by the ligand for CD 40 . J. Exp. Med. 178:669-674.

23. Melter, M., et al. 2000. Ligation of CD40 induces the expression of vascular endothelial growth factor by endothelial cells and monocytes and pro- motes angiogenesis in vivo. Blood. 96:3801-3808.

24. Caux, C., et al. 1994. Activation of human dendritic cells through CD40 cross-linking. J. Exp. Med. 180:1263-1272.

25. Dechanet, J., et al. 1997. CD40 ligand stimulates proinflammatory cytokine production by human endothelial cells. J. Immunol. 159:5640-5647.

26. Karmann, K., Hughes, C.C.W., Schechner,J., Fanslow, W.C., and Pober, A.S. 1995. CD40 on human endothelial cells: inducibility by cytokines and functional regulation of adhesion molecule expression. Proc. Natl. Acad. Sci. U. S. A. 92:4342-4346.

27. Bunescu, A., et al. 2002. Increases in circulating levels of monocyte-platelet and neutrophil-platelet complexes following hip arthroplasty. Clin. Sci. (Lond.). 102:279-286.

28. Terasaki, P.I., Cecka, J.M., Gjertson, D.W., and Takemoto, S. 1995. High survival rates of kidney transplants from spousal and living unrelated donors. N. Engl. J. Med. 333:333-336.

29. Bingaman, A.W., et al. 2000. Vigorous allograft rejection in the absence of danger. J. Immunol. 164:3065-3071.

30. Hancock, W.W., et al. 1996. Costimulatory function and expression of CD40 ligand, CD80, and CD86 in vascularized murine cardiac allograft rejection. Proc. Natl. Acad. Sci. U. S. A. 93:13967-13972.

31. Blair, P.J., et al. 2000. CD40 Ligand (CD154) triggers a short-term $\mathrm{CD} 4(+) \mathrm{T}$ cell activation response that results in secretion of immunomodulatory cytokines and apoptosis. J. Exp. Med. 191:651-660.

32. Larsen, C.P., Morris, P.J., and Austyn, J.M. 1990. Migration of dendritic leukocytes from cardiac allografts into host spleens. A novel pathway for initiation of rejection. J. Exp. Med. 171:307-314.

33. Boumpas, D.T., et al. 2003. A short course of BG9588 (anti-CD40 ligand antibody) improves serologic activity and decreases hematuria in patients with proliferative lupus glomerulonephritis. Arthritis Rheum. 48:719-727. 